\title{
Analysis on the Error of Time Synchronization
}

\author{
TENG Ling \\ Information Communication Department \\ China Electric Power Research Institute \\ Beijing 100192, China \\ tengling@epri.sgcc.com.cn \\ GAO Qiang \\ Information Communication Department \\ China Electric Power Research Institute \\ Beijing 100192, China \\ Gaoqiang2010@epri.sgcc.com.cn \\ WANG Yang \\ Information Communication Department \\ China Electric Power Research Institute \\ Beijing 100192, China
}

\author{
WANG Miaoxin \\ Information Communication Department \\ China Electric Power Research Institute \\ Beijing 100192, China \\ LAI Yuan \\ Telecommunication Company \\ State Grid Jibei Electric Power Co., Ltd \\ Beijing 100053, China \\ TIAN Yu \\ Telecommunication Company \\ State Grid Jibei Electric Power Co., Ltd \\ Beijing 100053, China
}

\begin{abstract}
In order to improve the NTP and PTP time synchronization accuracy, this paper summarizes the synchronization principle of two protocols. On this basis, the synchronization error sources are modeled one by one, and the error of the mathematical model are presented. Finally, experimental verification showed that the synchronization interval of NTP model is small, the local clock frequency deviation caused by the synchronization error is small, synchronous precision is high. Because the errors of the network protocol stack were eliminated, the synchronization accuracy of PTP is much higher than that of the NTP.
\end{abstract} model

Keywords—NTP; PTP; synchronization error; mathematical

\section{INTRODUCTION}

Network Time Protocol (NTP) is the most popular and most mature network time protocol. First proposed in 1985 by Professor David L. Mills Delaware University in the US, and the latest version is NTP v4, with synchronization accuracy of milliseconds ${ }^{[1]}$. It is time for the synchronous WAN computer to Internet standard protocol server time. At present, it is widely used in personal computers, and it is also applied to the financial, electronic commerce and other distributed systems that need to be accurate and reliable.

Precision Time Protocol (PTP) first appeared in 2002,the latest version was adopted in 2008 PTP v2, with submicrosecond synchronization accuracy ${ }^{[2]}$.

Currently, the principle of two synchronous Network Time Protocol NTP and PTP have been more comprehensive analysis $^{[3-6]}$, but the two kinds of synchronization protocol error sources and mathematical model are rarely researched.

\section{NTP SYNCHRONIZATION PRINCIPLE}

\section{A. NTP Synchronous mode}

There are three modes of NTP protocol, multicast mode, the client/server model, symmetric mode. Wherein the client/server model is the most typical way of working, the client/server mode synchronization model is a typical two-way time delay method ${ }^{[1]}$.In this mode, the client periodically sends NTP synchronization request packet to the server. Let $T_{i-3}$ is the message leaves the client when the timestamp and $T_{i-2}$ is the timestamp when the packet reaches the server. $T_{i-1}$ is the timestamp packets leave server. $T_{i}$ is the timestamp that is recorded by Client when the packet return back to the client

NTP algorithm is a typical two-way time delay method. Suppose the true clock skew (clock offset) between server A and Client B is $\theta_{i}$ at $T_{i}$ time, and the round-trip delay of NTP packets (roundtrip delay) is $\delta_{i}$, one-way path delay of packets from the client $\mathrm{B}$ to the server $\mathrm{A}$ is $\gamma$. One-way path delay of packets from the client $\mathrm{A}$ to the server $\mathrm{B}$ is $\beta$. According to the timing relationship between them and the four timestamps, you can get the following equation: 


$$
\begin{aligned}
& \theta_{i}=\frac{\left(T_{i-2}-T_{i-3}\right)+\left(T_{i-1}-T_{i}\right)+(\beta-\gamma)}{2} \\
& \delta_{i}=\gamma+\beta=\left(T_{i-2}-T_{i-3}\right)-\left(T_{i-1}-T_{i}\right)
\end{aligned}
$$

According to the clock offset $\theta_{i}$ value, client can adjust their own time, and synchronizes with the server.

\section{B. Error Analysis}

The synchronization accuracy of NTP is mainly limited by the following factors: the delay error introduced by the operating system protocol stack, network propagation delay and clock frequency error offset error introduced.

\section{1) Protocol stack error}

NTP is a protocol of application layer, the lower the use of transport layer protocol UDP port number is 123. Commonly used TCP/UDP Socket communication through programming, data prior to packaging, the network packet sent to the link through a series of calls and buffer, calls and buffering process introduces a timing error. This time the error introduced by Protocol stack is uncertain delay, real-time operating system and load-related, and real-time operating system load is a random process, can’t be predicted.

In order to reduce this error, kernel-level network packet filter can be used to achieve a one-time group calls and send multiple packets, maximum packet quickly send.

\section{2) Network propagation delay error}

NTP packets in the transmission process, not only has the inherent signal propagation delay, but also the router processing and queuing delay. Packet round trip may choose different routing paths, which will lead to round-trip propagation delay asymmetry signal; router queuing delay and instant network load-related. Can't be simply described by a stationary random process. These factors will ultimately lead to $\beta \neq \gamma$.

In the compute server A and Client B's clock offset formula (1), because $\beta$ and $\gamma$ can't be accurately measured and $\beta \neq \gamma$, and therefore unable to calculate the true value of the clock offset $\theta_{i}$, but you can calculate the clock skew interval in which the true value $\theta_{i}$.

Let

$$
\begin{gathered}
a=T_{i-2}-T_{i-3} \\
b=T_{i-1}-T_{i}
\end{gathered}
$$

In the actual calculation, approximate $\beta=\gamma$. According to the formula (1)-(4), the clock bias approximate:

$$
\theta=\frac{a+b}{2}
$$

$\theta_{\text {also is the middle point of }} \theta_{i}$. By the formula (5) can be drawn:

$$
\theta-\frac{\delta_{i}}{2} \leq \theta_{i} \leq \theta+\frac{\delta_{i}}{2}
$$

As can be seen, the maximum error instead of using approximations $\theta$ true value of $\theta_{i}$ to $\delta_{i} / 2$. If $\delta_{i}$ is smaller, instead of using approximations $\theta$ higher degree of confidence the true value of $\theta_{i}$. NTP time synchronization clock filter algorithm is based on this principle.

That clock filtered data filtering process for a time server, the purpose of treatment is to improve the delay estimation accuracy $\delta$ and $\theta$ clock offsets obtained through a network server. In the specific implementation, client retention n-th (usually take $\mathrm{n}=8)(\delta, \theta)$ value and put the value of $\theta$ corresponding to $\delta$ to process as the clock offset value recently obtained. After filtering algorithm using the clock offset can get 5 more accurately reflect the quality of the server clock.

\section{3) Clock frequency deviation} follows:

The local clock model of clients can be described as

$$
T(t)=T\left(t_{0}\right)+R\left(t_{0}\right)\left(t-t_{0}\right)+1 / 2 D\left(t_{0}\right)\left(t-t_{0}\right)^{2}+\varepsilon(t)
$$

$\mathrm{T}(\mathrm{T})$ is the time for the t time clock to display the time relative to the standard time. $D\left(t_{0}\right)$ is a drift of the local clock frequency in per unit time (the inverse of the frequency of the first order). $R\left(t_{0}\right)$ is the frequency of a local clock in the time of $t_{0} \cdot T\left(t_{0}\right)$ is the time of $t_{0}$ clock. $\varepsilon(t)$ random characteristics characterize the local clock. In a typical analysis, ignoring the second order term $1 / 2 D\left(t_{0}\right)\left(t-t_{0}\right)^{2}$.

By the formula (7), the time deviation between the client and the server is not only related to the $\theta$, but also to the deviation of the local clock frequency. In parallel with the basic clock synchronization must be calculated frequency deviation, or even the time to achieve the clock synchronization, after $\left(t-t_{0}\right)$,it will still be new clock error.

NTP does not get time to deal with deviation $T_{A}-T_{B}$ used directly to adjust the local clock, but to convert it to a control amount of the variable frequency oscillator through a loop filter to control the oscillation frequency, indirect 
adjustment system clock. By clock filtering and phase of the local clock/frequency prediction. NTP reduces the uncertainty of the network delay jitter the frequency offset of the local clock is compensated, and the synchronization precision is improved.

In the formula (7), A (that is the client to issue two adjacent intervals of time synchronization request to the server), the more the value of the first-order and second-order term, the greater the precision of the corresponding synchronization will be reduced. The NTP time servers and ordinary computer were used to verify this conclusion.

Set the time server to work in client/server mode, the computer A synchronous packet transmission interval is set to 16 s, computer B synchronization packet interval before and after the two tests are set to $64 \mathrm{~s}$ and $256 \mathrm{~s}$.

Test results are shown in table 1.

TABLE I. THE TEST RESULTS OF NTP

\begin{tabular}{|c|c|c|c|}
\hline Object & Time/h & Mean/ms & $\begin{array}{c}\text { standard } \\
\text { deviation/ms }\end{array}$ \\
\hline Computer A & 16 & 0.07 & 0.16 \\
\hline Computer B & first 8 & 1.81 & 1.61 \\
\hline Computer B & last 16 & 8.67 & 3.63 \\
\hline
\end{tabular}

In table 1, we can see that since the computer there is a deviation of the local clock frequency, extend the synchronization interval will reduce the time synchronization accuracy.

\section{PTP SYNCHRONIZATION PRINCIPLE}

\section{A. PTP synchronization pattern}

PTP between the master clock and slave clock synchronization through periodic exchange of messages to be completed. Every packet switching slave clock can get four timestamps. Through these four timestamps, slave clock can be calculated from the master clock offset and path delay between clocks .

Compared with NTP, one of the advantages of PTP is that it generate timestamps through the physical layer, which can eliminate the delay caused by packet processed and improve the accuracy of the synchronization.

\section{B. PTP error analysis}

PTP due to the use of hardware-assisted acquisition of the timestamp method, eliminating the network protocol stack delay jitter introduced by the synchronization error, which is the biggest difference between the PTP and NTP. NTP is similar except that the main source of error is the PTP synchronization and network components from the clock itself.

\section{1) Network components}

The accuracy of sub-s synchronization of PTP must be compensated for the propagation delay of network components. Specific implementations, support PTP protocol transparent clock replace the ordinary router or switch. Transparent clock can accurately measure PTP packet network components residence time and the residence time in the recording PTP packet, PTP synchronization algorithm utilizes the residence time of the delay introduced by the network components to compensate, improving PTP synchronization accuracy.

\section{2) Slave clock frequency deviation}

Slave clock frequency characteristic must be consistent with the requirements of the synchronization interval and accuracy specifications. Measurement Uncertainty PTP hardware timestamp in the order of tens of nanoseconds. The frequency offset from the clock itself is the main source of error.

PTP implementation is sub-microsecond synchronization accuracy, so choose a low frequency deviation, frequency drift small crystal oscillator for PTP is very important. No ordinary temperature compensated crystal oscillator frequency deviation of up to $5 \times 10^{-5}$ in the whole temperature range with temperature compensated crystal oscillator frequency deviation of up to $2.5 \times 10^{-6}$, and inexpensive quartz oscillator frequency deviation of up to $1 \times 10^{-4}$.

In order to reduce application costs, in computer applications PTP protocol, allows the use of ordinary cheap crystal oscillator. For frequency deviation $1 \times 10^{-4}$, will produce in $1 \mathrm{~s}$ intervals of time deviation $100 \mu \mathrm{s}$, to achieve synchronization accuracy of less than $1 \mu \mathrm{s}$, the frequency deviation of the local clock must be less than $1 \times 10^{-6}$, visible directly using ordinary the quartz crystal oscillator cannot meet the requirements must be taken frequency compensation techniques.

In the application, the local clock calculates the frequency offset of the time to reach the time of continuous multiple Sync packets, and then compensates the frequency offset. Frequency compensation techniques can use digital compensation technology, the prevailing practice is to use PI regulation technology.

\section{Verification of Sub-microsecond synchronization accuracy}

In the lab, the master clock is connected to the slave clock through the PTP switch, and the relative accuracy of the master clock and the slave clock is tested by the time interval analyzer through the clock's 1PPS output. The connection diagram shown in Figure 1.

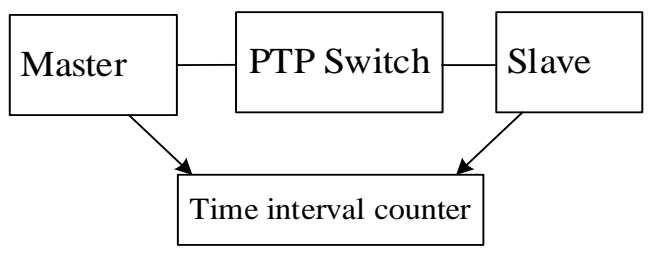

Fig. 1. synchronization system connection diagram

The PTP switch is set to P2P transparent clock. Synchronization test duration half an hour. The average is the 43.7ns, the standard deviation is $21.9 \mathrm{~ns}$, which reached the sub-microsecond synchronization accuracy. 


\section{COMPARISON OF TWO PROTOCOLS}

NTP and PTP as two different network time synchronization protocol, there are similarities but also differences.

NTP client/server mode and the PTP master-slave synchronization mechanism is similar pattern. Both by the client and the server (master clock and the slave clock) messages exchanged between the four timestamps to obtain information, and to calculate the local clock is offset and path delay. In this similarity under, NTP and PTP also has details on the differences between the specific implementation .First, on a timestamp mark, NTP timestamp using the software, PTP hardware time stamp, which is the biggest difference between the two protocols. Secondly, the initiator NTP synchronization process is the client (server/client mode), the initiator is the PTP master clock synchronization process. Thirdly, update frequency synchronization NTP configuration by the user, the minimum 16s, default 64s.PTP default update frequency 2 s, can be smaller. With respect to the NTP synchronization millisecond precision, hardware timestamps and higher update frequency PTP allows PTP synchronization with submicrosecond precision.

NTP and PTP belong to the network time synchronization protocol, but they are not the same. NTP is suitable for tens of thousands of computers, but PTP is suitable for time synchronization of LAN nodes. The extension scope use of PTP WAN is currently not realistic: First, PTP clients need hardware timestamp support and network components, extend to a huge WAN costs. Second, synchronization packets from the asymmetric delay uncertainty path and network components will be introduced to reduce the spread within the WAN when PTP synchronization accuracy, can't meet the accuracy requirements of sub-microsecond synchronization.

Finally, NTP management needs user configuration, and PTP is adaptive management. Table 1 is a comparison of the main differences and similarities of NTP and PTP.

\section{CONCLUSION}

The synchronization error of NTP and PTP protocols mainly come from the network component and the local clock frequency offset. NTP uses the clock filtering algorithm to reduce the error of the network component delay, while the PTP uses a transparent clock to compensate the delay of the network components. The two protocols need to use the frequency compensation technique to overcome the error that introduced by the local clock frequency difference. PTP time synchronization accuracy is better than NTP, but its application is limited to LAN, how to expand the application scope of PTP need to be further studied.

\section{ACKNOWLEDGMENT}

This work is supported by the Science and technology project of State Grid Corporation of China (SGCC) "Research and application of the key technology of power synchronization based on time frequency fusion”.

\section{REFERENCES}

[1] YANG Xian-jie. Research and Application of NTP Protocols[J]. Electric Power IT.2011,9(6):28-32.

[2] John C. Eidson.IEEEStandard for a PrecisionClock Synchronization Protocol forNetworked Measurement and ControlSystems[S]. IEEE. 2008.

[3] Teng Ling,Gao Qiang,Wang Yang,Zhang Xiaomei.Feasibility Research on Integration of Power Frequency Synchronization Network With Time Synchronization Network[J].Power System Technology,2015,39(1):294298.

[4] Cui Quansheng,Wei Yong,He Yongji,et al.Analysis of PTP 1588 protocol[J].Power System Protection and Control,2011,39(10):148154(in Chinese).

[5] Huang Shu,Cui Quansheng,Song Xiaohui,Di Junfeng. Study of IEEE 1588 Conformance Testing in Electric Power System[J].Power System Technology,2012,36(6):89-94

[6] Hu Yongchun,Zhang Xuesong,Xu Weiguo,et al.Error analysis and detection method for IEEE1588 clock synchronization system[J].Automation of Electric Power Systems,2010,34(21):107111(in Chinese). 